\title{
A participação de stakeholders no planeamento e gestão do turismo e da recreação em áreas protegidas: Fundamentos sobre o processo participativo
}

\author{
Stakeholders participation in tourism and recreation planning and management in protected areas: Fundamentals on the \\ participatory process
}

\section{Paulo Filipe Rosa}

Sport Sciences School of Rio Maior, Polytechnic Institute of Santarém and Centre of Tourism Applied Research (CiTUR), Avenida Dr. Mário Soares, nr. 110, 2040-413 Rio Maior, Portugal, prosa@esdrm.ipsantarem.pt

\section{Luís Alberto Dias Carvalhinho}

Sport Sciences School of Rio Maior, Polytechnic Institute of Santarém and Centre of Tourism Applied Research (CiTUR), Avenida Dr. Mário Soares, nr. 110, 2040-413 Rio Maior, Portugal, Icarvalhinho@esdrm.ipsantarem.pt

\section{Jorge Alexandre Pereira Soares}

University of Madeira, Colégio dos Jesuítas - Rua dos Ferreiros 9000-082 Funchal, Portugal, jorges@staff.uma.pt

\section{Resumo}

O turismo e a recreação em áreas protegidas têm vindo a tornar-se cada vez mais relevantes a nível internacional e nacional. Neste sentido, é importante que estas áreas assumam princípios de planeamento e gestão destas práticas, de forma a melhor se enquadrarem neste crescimento e contribuírem para o desenvolvimento sustentável dos territórios onde se inserem. Neste domínio, surge a gestão participativa como uma condição essencial para o alcance dos princípios da sustentabilidade. Este artigo reflete um trabalho de revisão de literatura, com vista a uma melhor compreensão dos fundamentos que regem estes processos. A reflexão daqui derivante realça a importância de, a par de aspetos processuais, se considerar de forma mais aprofundada um conhecimento do perfil dos stakeholders, assente na comunicação ativa e legitimação para a participação. Considera-se que um conhecimento adequado do seu perfil e perspetivas acerca dos vários domínios do processo, poderá reduzir os problemas mais comuns derivantes da sua implementação.

Palavras-chave: Turismo, recreação, áreas protegidas, stakeholders, processos participativos.

\section{Abstract}

Tourism and recreation in protected areas is becoming increasingly relevant at an international and national level. Therefore, it is important that these areas adopt principles of planning and management of tourism and recreation practices in order to better fit in this growth and contribute to the sustainable development of the territories. Regarding management, we found participatory management as an essential condition for the achievement of sustainability principles. This article reflects a literature review regarding a better understanding of the fundamentals governing these processes. The reflections made in this article stress, along with procedural aspects, the importance of a profound understanding of the stakeholders profile, based on active communication and legitimacy to participate. It considers that an adequate understanding of the stakeholders profile and perspectives about the several factors of the management process may reduce the most common problems deriving from its implementation.

Keywords: Tourism, recreation, protected areas, stakeholders, participatory processes.

\section{Introdução}

No contexto das Áreas Protegidas (AP), o turismo e a recreação surgem como dois dos vários setores de atividade que têm efetuado maior uso do território (Walpole \& Goodwin, 2001). No entanto, a existência de AP, aliada a outras potencialidades físicas do território não é suficiente para garantir a sustentabilidade destas atividades. Neste contexto, Dearden, Bennett e Johnston (2005) referem que "Não é suficiente ter o número certo de AP, nos locais certos, é igualmente necessário assegurar que a sua governança é capaz de efetuar uma gestão efetiva de forma a produzir os resultados esperados". A governança é o processo pelo qual as sociedades ou organizações constituem a suas decisões importantes, determinam aqueles que envolvem nesse processo e a forma como prestam contas. Esta envolve o Estado mas transcende-o no sentido em que envolve corporações, organizações não-governamentais e indivíduos (Eagles, 2009), ou seja, está associada ao envolvimento de outras instituições e indivíduos numa perspetiva participativa, quer ao nível do planeamento, quer da gestão. Neste sentido, governança e gestão são aspetos inerentes ao desenvolvimento do turismo e da recreação nas AP.

São várias as estruturas de gestão características das AP cuja determinação e adequabilidade advém de características próprias da área, da região e do país onde estão estabelecidas. Neste âmbito, principalmente a partir de meados dos anos 80 , uma nova forma de entender a gestão emerge entre a comunidade científica. Referimo-nos à gestão participativa, ou colaborativa, cujos princípios se regem pela participação pública de todos aqueles que de alguma forma estão envolvidos e são afetados pelas decisões de gestão afetas às AP. Seja por razões morais, políticas ou de eficiência, estes processos tendem a aumentar um pouco por todo o Mundo.

Aqueles que participam são denominados de stakeholders (em português, "partes interessadas"). De acordo com Gray (1985) um stakeholder é aquele que tem o direito e a capacidade de participar num processo. Um stakeholder que é impactado pela ação de outro tem o direito de ser envolvido; um stakeholder deve ter um conjunto de competências e conhecimentos. Neste contexto, um stakeholder no setor do turismo é alguém que é impactado pelo desenvolvimento do turismo de forma positiva ou negativa. A participação ativa de stakeholders nos processos de planeamento e gestão são característicos de diversas áreas da sociedade, com destaque ao nível do planeamento urbano e regional, ao nível da conservação da natureza e, por estar diretamente ligado com a última, ao nível do turismo sustentável, desenvolvido em áreas rurais ou naturais. 
Os processos participativos regem-se por um conjunto de fundamentos e são caracterizados por benefícios que afetam a generalidade dos stakeholders e, ao mesmo tempo, por barreiras e dificuldades à sua implementação. Uma das principais barreiras e de mais difícil resolução prende-se com a legitimação dos stakeholders para participar. Uma segunda, prende-se com os conflitos que se podem gerar entre os stakeholders ou entre estes e as estruturas responsáveis pelo planeamento e gestão. No entanto, mesmo conscientes dos problemas, é claramente assumido na investigação científica, a pertinência da participação de stakeholders nas várias fases de planeamento e gestão do turismo em AP, sobretudo ao nível da recolha de informação e posteriormente no apoio à tomada de decisão e implementação (Jamal \& Getz, 1995; Patti, 2013; Wray, 2011; Yodsuwan \& Butcher, 2012).

É com base nestas características e com o intuito de explorar os princípios teóricos que fundamentam estes processos que desenvolvemos o presente trabalho de revisão de literatura. Reconhecemos primeiramente que em Portugal, os trabalhos de natureza científica nestes domínios têm sido reduzidos. Igualmente reconhecemos que o planeamento e gestão do próprio fenómeno turístico e recreativo no seio das AP portuguesas não têm sido aspetos prioritários, sendo alvo de recomendações em diversos trabalhos académicos sustentados por dados empíricos (Laranjo, 2011; Rosa, 2014). Em segundo, considerando ainda o caso nacional, verifica-se igualmente que a participação ativa de stakeholders em diferentes domínios respeitantes ao território protegido é um assunto pouco explorado na literatura nacional e por isso, com consequências na sua aplicação prática (Rosa, 2014).

Com o intuito de promover um primeiro contributo para a melhoria da investigação e implementação destes processos em Portugal associam-se alguns trabalhos que marcaram a teoria ainda hoje vigente com assunções de alguns estudos mais recentes. Inicialmente são apresentados fundamentos associados a este paradigma de gestão, sendo estes seguidos de uma descrição mais detalhada sobre os benefícios e problemas relacionados com a implementação destes processos. A reflexão decorrente deste ponto leva-nos posteriormente a analisar questões de envolvimento e legitimação para a participação como essenciais para o sucesso. Concluímos este trabalho, apresentando um conjunto de princípios teóricos e respetivas ligações entre si, que devem reger as considerações associadas à iniciação de processos de gestão desta natureza.

Desta forma, visamos um aprofundamento e sistematização da teoria e um contributo para uma determinação mais consciente e fundamentada da iniciação de processos participativos em AP considerando a articulação com os fenómenos turísticos e recreativos.

\section{Fundamentos de um paradigma de gestão em AP: a gestão participativa e integrada}

A par do crescimento do turismo enquanto indústria global e da sua reconhecida importância para as economias de muitas nações mundiais, surgem também muitas críticas acerca deste setor económico pelos impactos negativos a nível económico, ambiental e sociocultural, evidentes em muitos destinos (Ladkin \&
Bertramini, 2002). Estes criticismos deram origem a uma maior sensibilização para os seus efeitos negativos e até para novas aproximações de gestão dos espaços recetores, desenvolvidas muitas vezes a curto prazo e sem ter em conta fatores que influenciam o desenvolvimento sustentável. Neste seguimento, algumas aproximações de planeamento e gestão contemporâneas, como o desenvolvimento sustentável, planeamento integrado, turismo de base comunitária, entre outros, têm tentado sustentar o turismo como um veículo para o desenvolvimento sociocultural e económico (Tosun \& Jenkins, 1998).

No contexto das AP, enquanto espaços de usufruto turístico e recreativo, também se verifica que as tendências na gestão têm vindo a sofrer grandes alterações (Kalamandeen \& Gillson, 2007; Pinto, 2008). Novos paradigmas emergem entre as comunidades científicas e profissionais com o intuito de melhorar e adaptar de forma cada vez mais eficaz, os processos de gestão inerentes a estes espaços, onde a proteção da natureza e dos recursos culturais são fatores basais.

Contudo, e de forma contrária a este princípio, um dos claros problemas que advém da implementação das AP está diretamente relacionado com as populações locais dessas mesmas áreas, que outrora (antes da criação da AP), desenvolveram as suas atividades socioeconómicas de forma livre e organizadas segundo padrões de gestão tradicionais e que incluem organizações sociais tradicionais para gestão de zonas de caça, pesca, artefactos religiosos, entre outros (Borrini-Feyerabend et al., 2013) . De acordo com Pimbert e Pretty (1995), no passado, algumas populações locais, após a implementação de determinados espaços protegidos, foram "expulsas" dos seus habitats naturais sem o provisionamento adequado de fontes de rendimento/trabalho. Noutros casos, e mais recentemente, determinadas populações têm vindo a sofrer restrições perante a utilização dos espaços (uso comum da propriedade) para o desenvolvimento de atividades associadas ao meio natural (e.g. agricultura). De acordo com os mesmos autores, o descontentamento derivado destas situações pode levar a um risco crescente de conflitos no meio rural e à consequente destruição daquilo que se pretendia ver protegido. Protestos, ataques, incêndios, entre outros, são apenas alguns exemplos de situações que poderão acontecer derivadas do desagrado das populações locais relativamente à implementação das AP e consequentemente às decisões de gestão a elas inerentes.

Igualmente importante é o fato de se continuar a ignorar as instituições locais e organizações sociais presentes numa determinada AP. Como resultado, sistemas locais de tomada de decisão e de gestão de recursos são desconsiderados e as instituições locais são substituídas por corpos profissionais e burocráticos, quando na realidade, as primeiras são cruciais para a conservação e desenvolvimento sustentáveis do uso da biodiversidade, para além de que são recursos que devem ser potenciados, alterados e desenvolvidos e não ignorados e suprimidos (Pimbert \& Pretty, 1995).

Mediante estes factos, e tendo em conta que a sociedade se torna cada vez mais complexa e as economias mais interdependentes, diversas organizações têm encontrado uma dificuldade crescente 
em agir de forma unilateral para atingir determinados objetivos (Selin, 1999). É neste campo, que a participação e o envolvimento público têm vindo a ser defendidos como uma tendência em crescendo dentro do planeamento urbano e regional e na tomada de decisão, embora em determinados aspetos, identificada como problemática na prática (Wray, 2011).

Os processos participativos têm um grande relevo ao nível da conservação da natureza. Khadka e Nepal (2010) defendem que a conservação tem vindo a sofrer uma alteração profunda na sua filosofia, políticas e aproximações de gestão ao longo dos últimos 40 anos. A aproximação tradicional (Top-Down management) tem vindo a ser criticada por falhar ao incluir elementos sociais críticos nas práticas de gestão e está a ser gradualmente conjugada com estratégias participativas (Bottom-up). No mesmo sentido que os autores anteriores, Wray (2011), referindo-se ao setor do turismo, defende que a participação pública se tornou um princípio fundamental da sustentabilidade e responsabilidade social, e sugere que, ao olharmos para as comunidades envolvidas no turismo, é impossível separar os processos sociais, económicos e políticos que operam dentro de uma comunidade.

Destas conceções de participação, emerge a noção de gestão adaptativa. Segundo Stringer et al. (2006), esta trata o conhecimento acerca dos ecossistemas como incertos e plurais, partindo do princípio que, para criar estratégias de desenvolvimento sustentável é necessária a participação de stakeholders e a constituição de relações entre estes, num processo contínuo de alteração, adaptação e aprendizagem. De todas estas, o fator aprendizagem é dos mais importantes e valorizados, refletindo-se no espelho da aprendizagem social, uma aproximação que, segundo Stringer et al. (2006) reflete muito do trabalho realizado pelo conhecido psicólogo Albert Bandura. Esta é essencial nos processos de gestão adaptativa dada a sua dinâmica.

De acordo com Pimbert e Pretty (1995), na história da participação comunitária, duas escolas de pensamento e prática têm sido desenvolvidas: Uma vê a participação comunitária como um meio para aumentar a eficiência. A ideia central é de que, se as pessoas forem envolvidas, então estas estão mais satisfeitas e de acordo para suportar a inovação e desenvolvimento de um determinado conjunto de serviços ou processos; A segunda vê a participação comunitária como um direito, cujo principal objetivo é o início da mobilização para uma ação coletiva e construção de uma instituição.

Na realidade, as duas visões têm uma perspetiva inclusiva, seja por motivos de melhoria de processos seja por motivos morais, e assim sendo, ambas assentam num quadro com traços teóricos comuns.

O trabalho de (Gray, 1985, 1989, 2004) é emblemático ao nível da investigação em colaboração. Este define a colaboração como um processo de decisão conjunto entre os diferentes stakeholders, relativo a um determinado problema pertencente a um domínio, acerca do futuro desse mesmo domínio. São identificadas cinco características críticas do processo colaborativo: (1) Os stakeholders são interdependentes; (2) As soluções emergem se lidarmos de forma construtiva com as diferenças; (3) A propriedade das decisões deve ser conjunta; (4) Os stakeholders assumem coletivamente a responsabilidade para a direção futura de determinado domínio; (5) A colaboração é um processo emergente e dinâmico (Gray, 1989; Selin, 1999).

Segundo Selin (1999), compreendendo as diversas formas possíveis de parceria dadas em resposta às diferentes pressões sociais, os gestores do turismo podem iniciar a constituição de parcerias que promovam as respostas mais apropriadas para resolver problemas de foro interativo ou tomar vantagem em oportunidades emergentes. Um exemplo claro que emerge deste trabalho é que a forma de potenciar o envolvimento público e suporte para a existência de parcerias advém da possibilidade de envolver as pessoas nos processos de planeamento prévio. Para Selin (1999)a lição mais importante para os gestores do turismo é, provavelmente, que as parcerias e a colaboração são processos dinâmicos e não um fenómeno estático.

Ao longo das últimas três décadas, o declínio de muitas indústrias tradicionais e rurais, como a agricultura ou a atividade mineira, levou a que muitas comunidades rurais explorassem indústrias alternativas para fortalecer as suas economias de base (Byrd, 2003). O Turismo tornou-se assim uma das grandes indústrias com potencial para a diversificação e desenvolvimento económico das comunidades sobretudo a nível rural em espaços protegidos, e por isso, um dos objetivos das entidades que gerem estas áreas, deve portanto, consistir num maior envolvimento, capacitação e mobilização de diversas pessoas e grupos.

\section{Fatores para a implementação de sistemas participativos}

Numa primeira instância, a literatura apresenta considerações que antecedem estes processos, nomeadamente um conjunto de condições (entendam-se, pressupostos importantes para a efetividade destes processos) e simultaneamente, fatores inibidores (entendam-se aspetos que dificultam a sua implementação e desenvolvimento). Numa segunda, é possível identificar aspetos que resultam destes processos, nomeadamente benefícios (aspetos positivos derivantes da sua implementação) e problemas (aspetos negativos derivantes da sua implementação).

É sobre estes fatores e dois momentos (Pré processo e durante) que nos centramos seguidamente.

\subsection{Condições vs inibidores}

O sucesso dos esforços para gerar um processo participativo de planeamento e gestão depende de uma variedade de fatores. Este vai depender não apenas das estruturas formais em que é estabelecido mas também das motivações, personalidades e perceção das regras/funções dos vários envolvidos (Ladkin \& Bertramini, 2002). Para isso, os responsáveis por este processo devem recolher opiniões e perspetivas de todos os stakeholders interessados que estão envolvidos nos processos de decisão relacionados com o planeamento (Byrd, 2007). Num sentido concordante, Wray (2011) explica que quando as comunidades são ativamente envolvidas nos processos de planeamento e implementação, os planos tendem a ser mais afetos às necessidades e expetativas dos stakeholders, motivando-os a alcançar benefícios económicos, sociais e ecológicos. 
Jamal e Getz (1995) apresentam seis fatores críticos para o sucesso do trabalho em parceria: 1) O reconhecimento de que um alto grau de interdependência no planeamento e na gestão de determinado projeto é uma realidade; 2) O reconhecimento dos benefícios individuais/mútuos que podem derivar do processo colaborativo; 3) A perceção de que as decisões são para ser implementadas; 4) A inclusão de grupos chaves de stakeholders; 5) A nomeação de um responsável legítimo para iniciar e facilitar o processo de colaboração; 6) Formulação clara de metas e objetivos.

Outro aspeto a ter em conta, relaciona-se com um conhecimento adequado do contexto (local, regional ou nacional) em que a participação se desenvolve. As evidências sugerem que o planeamento colaborativo é diretamente afetado por regras, práticas e sistemas que diferem entre nações, regiões e locais (Aas, Ladkin, \& Fletcher, 2005; Roberts \& Simpson, 1999). Em concordância, Bramwell e Lane (2000) defendem que algumas aproximações colaborativas que funcionam em alguns países ocidentais muito desenvolvidos podem não funcionar noutros em estágios económicos e políticos diferentes ou menos desenvolvidos.

Em complementaridade às condições apresentadas, são referidos aspetos que de alguma forma inibem/dificultam a implementação destes processos. Por um lado, estas dificuldades podem ser colocadas pelo Estado. Pimbert e Pretty (1995) referem que, mesmo reconhecendo a importância da participação, muitos profissionais de conservação colocam limites muito claros na forma e grau de participação que toleram na gestão das AP. Isto significa que as agências de gestão (maioritariamente estatais) precisam e receiam a participação popular, ou seja, necessitam da concordância e apoio das pessoas mas têm medo que este maior envolvimento seja menos controlável, menos preciso e assim torne todos os processos de planeamento mais lentos. Os mesmos autores referem ainda que a participação total das comunidades locais no planeamento e gestão de AP pode ainda ser difícil no sentido em que é geralmente assumido que estas são, e devem ser, sujeitas a regimes de gestão externa (e.g. somente pelo Estado ou pelo Estado em parceria com ONG's internacionais com especialistas ao nível da conservação).

Para além destes, outros inibidores têm vindo a ser identificados, como por exemplo: 1) em países em que o turismo se depara com dificuldades de planeamento e gestão, a colaboração pode ser difícil de atingir (Aas et al., 2005); 2) Fatores relacionais podem também desencorajar a colaboração entre stakeholders ou até, quando existe diferenças no poder das diferentes partes envolvidas (Selin \& Chavez, 1995).

Ladkin e Bertramini (2002) acrescentam, referindo alguns dos inibidores mais significantes à participação: 1) a falta de especialistas e conhecimento ao nível das autoridades que planeiam o turismo; 2) Tradições politicas que favorecem a centralização da autoridade; 3) Falta de fundos; 4) Falta de interesse ou compromisso de stakeholders; 5) Competição pelos mesmos recursos; 6) Falta de estratégias a longo prazo e a 7) Falta de consenso em estruturas e processos específicos. Wray (2011) reforça estas assunções, referindo que a extensão/dimensão da participação comunitária é também fortemente afetada pelos 8) recursos financeiros disponíveis a nível local; 9) A relação cultural das comunidades recetoras com o comércio do turismo; 10) Níveis de experiência (dos stakeholders e entidades responsáveis pelo desenvolvimento do processo) no turismo e especialistas e competências no turismo local.

\subsection{Benefícios vs problemas}

Bramwell e Lane (2000) apresentam um sumário dos potenciais benefícios da colaboração no planeamento turístico e que incluem: 1) a providência de soluções de baixo custo em determinadas regiões, por 2) agrupamento de recursos evitando os custos de potenciais conflitos de interessados; Politicamente, o 3) processo colaborativo é mais legítimo e equitativo que as aproximações convencionais de planeamento, visto que 4) promove a interação, partilha e participação, em que os pareceres e recomendações de não-atores da indústria são tão legítimas como as de um expert.

Também Garrod (2003) sugere alguns benefícios, tais como: 1) Aumento da eficiência de projetos pela consulta à população local ou envolvendo-os na gestão e implementação dos mesmos; 2) Aumento da eficácia dos processos através de um maior envolvimento local para ajudar a garantir que os objetivos deste são cumpridos e os benefícios são recebidos pelo grupo pretendido; 3) Reforço das capacidades dos beneficiários para entender o que é o ecoturismo e como é que este pode contribuir para o desenvolvimento sustentável (por garantir que os participantes estão envolvidos ativamente no projeto através de formação formal e/ou informal e ações de sensibilização); 4) Aumenta a capacitação local, procurando dar às comunidades um maior controlo sobre os seus recursos e as decisões relativas à utilização dos mesmos e que afetam as suas vidas (o que significa assegurar que as comunidades locais recebem os benefícios associados com o uso desses recursos); 5) Compartilha os custos com os beneficiários locais, por exemplo, os custos de financiamento, operação, manutenção, de acompanhamento do projeto e os custos de avaliação.

Além disso, a colaboração adere valores às políticas vigentes, visto que as pessoas que são afetadas pelo desenvolvimento trazem o seu conhecimento e experiências ao processo. $O$ carácter inclusivo das aproximações colaborativas podem portanto ajudar a lidar com alguns problemas chaves da implementação de modelos de gestão, como os conflitos que surgem entre políticas e valores, ou interesses dentro da estrutura que implementa o processo (Hall, 2000).

Contrariamente a esta visão positiva, são também apresentados problemas relacionados com o processo em si. Garrod (2003), ao desenvolver acerca do trabalho de Drake (1991), referencia alguns problemas, nomeadamente: (1) A gestão da participação local, frequentemente implica 0 aumento do staff para o desenvolvimento do projeto; (2) É muitas vezes exercida alguma pressão pela comunidade para alargar o âmbito ou a forma do projeto além do que estava inicialmente planeado com consequentes aumentos de custos; (3) Os responsáveis pelo planeamento correm o risco de perder o projeto para forças opostas que têm interesse em assumir o controlo do mesmo longe da agência que o executa; (4) Os benefícios podem nem sempre 
alcançar todos os envolvidos e da forma esperada; (5) A informação das populações locais acerca de determinado projeto pode provocar o aumento das suas aspirações ao mesmo, levando a uma maior insatisfação ou frustração se o projeto falhar ou se atrasar; (6) Tentativas de envolver a comunidade local podem trazer alguns conflitos latentes que servem como entrave à implementação do projeto.

Swarbrooke (1999) por sua vez apresenta também algumas desvantagens: 1) Os processos participativos podem aumentar os custos do planeamento e desenvolvimento do turismo; 2) 0 envolvimento da comunidade local pode aumentar o período de tempo necessário para trazer projetos mais complexos à concretização; 3) Pode ser dada a oportunidade a grupos de interesse de negar às pessoas de fora da área em causa, oportunidades de emprego ou de lazer; 4) Pode permitir a grupos comunitários maioritários a descriminação contra outros (e.g. minorias étnicas).

Também Timothy (1999) numa perspetiva ligeiramente diferente, apresenta alguns constrangimentos: 1) Em muitas sociedades, existe uma cultura de respeito para com pessoas em posições de autoridade. Os que assumem estas posições (de autoridade) não têm necessariamente que procurar o consentimento daqueles que estão sob sua liderança, enquanto que aqueles que não têm autoridade, esperam para serem conduzidos/liderados. 0 planeamento é visto como algo que aqueles em posições de liderança fazem para o benefício daqueles que estão sob sua autoridade; 2) Embora muitas vezes possa ser mais eficaz do que outras abordagens para o planeamento e gestão do ecoturismo, o planeamento participativo pode também ser mais caro. Nas situações em que os orçamentos das autarquias locais estão sujeitos a severos cortes, o planeamento participativo pode ser simplesmente um luxo que as autoridades competentes sentem que não podem pagar; 3) Ao mesmo tempo, a população local pode sentir que não tem possibilidades de investir a tempo útil de participar no processo de planeamento. Em tempos de dificuldades económicas (que é ironicamente quando as iniciativas de ecoturismo são muitas vezes mais necessárias), as pessoas locais tendem a estar mais interessadas em fazer face às despesas do presente ao invés de planeamento para o futuro; 4) Falta de formação e especialização por parte dos responsáveis pelo desenvolvimento dos processos participativos também podem agir como um obstáculo para o desenvolvimento do turismo. Como resultado, as autoridades de planeamento muitas vezes sentem-se mal preparadas para se envolverem numa abordagem participativa, preferindo em vez disso, manter o uso de técnicas testadas e confiáveis; 5) Pode haver uma falta de compreensão crítica pela população local acerca da conceção do que é o turismo (e as suas vertentes). Membros da estrutura responsável tendem a ser por vezes relutantes em permitir que a comunidade possa participar porque duvidam da qualidade e utilidade de muitas informações. Ao mesmo tempo, a população local também não está disposta a envolver-se no planeamento, porque se sentem incapazes de lidar com as questões envolvidas e não são encorajados a acreditar que têm algo valioso para contribuir.

Apesar de nem todos os aspetos referidos anteriormente estarem presentes num determinado local e tempo, individualmente ou coletivamente estes representam vantagens e desvantagens significativas na implementação de uma aproximação colaborativa.

Se por um lado é amplamente defendido que as perceções dos benefícios positivos da colaboração facilitam a interação entre stakeholders, tão ou mais importante é a perceção das condições, inibidores e problemas à participação. Por isso mesmo, a formulação conjunta de metas e objetivos no planeamento do turismo e da recreação devem ser tomadas desde o início deste processo. É a vontade de lutar por um bem comum que se torna uma precondição essencial para o desenvolvimento de uma aproximação colaborativa.

Mediante as referências a estes fatores, apresentamos a figura seguinte (figura 1) que, de forma esquemática, representa um resumo das várias assunções anteriormente apresentadas.

$\mathrm{Na}$ figura podemos observar os fundamentos que regem a pertinência e importância dos processos de gestão participativos. De igual forma, e mediante as leituras realizadas, apresentamos um conjunto de condições e inibidores, assim como, de benefícios e problemas relacionados com a sua implementação.

O início do processo deve passar pela compreensão da realidade inerente ao maior número possível de condições e inibidores de forma a garantir que estas são controladas pelos responsáveis de determinado projeto.

Apesar de alguns dos problemas serem praticamente inevitáveis, parece-nos claro que a maximização dos benefícios implica consequentemente uma mitigação dos outros fatores.

Face à generalidade da figura apresentada, dois aspetos são essenciais: Em primeiro lugar, o papel dos responsáveis pela implementação destes processos é determinante, onde a experiência e competência no planeamento da recreação e do turismo, aliadas à personalidade dinâmica, capacidade de diálogo e compreensão mútua poderão garantir parte do sucesso. Em segundo, a consciência clara de quem são os stakeholders e a legitimidade que têm em participar no processo.

Pela denotada importância atribuída à consideração prévia de um conjunto de inibidores/condições, considerando que estas considerações, se tidas em conta previamente (i.e. antes do próprio processo em si) podem ditar o sucesso de um processo de gestão participativo (Rosa,2014) e que, envolvendo pessoas (stakeholders), estão intimamente dependentes das suas características (Donaldson \& Preston, 1995; Gray, 2004; Ladkin \& Bertramini, 2002), consideramos pertinente aprofundar no ponto seguinte, alguns fundamentos relacionados com o envolvimento e legitimação de stakeholders para a participação. 
Figura 1 - Processos de gestão participativos

\begin{tabular}{|c|c|}
\hline $\begin{array}{c}\text { Processos de gestão } \\
\text { participativos }\end{array}$ & \begin{tabular}{l}
\multicolumn{1}{c}{ Fundamentos } \\
- Contraria a insustentabilidade \\
- Tende para projeções a médio-longo \\
prazo
\end{tabular} \\
\hline & $\begin{array}{l}\text { - Aproximação "Bottom-up" } \\
\text { - Conjuga processos sociais, económicos } \\
\text {, políticos e ambientais } \\
\text { - Promove a satisfação e aceitação social } \\
\text { - Construção de uma instituição } \\
\text { (mobilização e fortificação social } \\
\text { - Processo emergente } \\
\text { - Promover aprendizagem social } \\
\text { - Dever moral } \\
\text { - Ponto com gestão adaptativa }\end{array}$ \\
\hline
\end{tabular}

Devem ser tidos em conta antes do início do processo

\begin{tabular}{|c|c|}
\hline \begin{tabular}{l}
\multicolumn{1}{c|}{ Condições } \\
- Envolvimento de todos \\
- Perceção das regras por todos \\
- Reconhecimento de um problema \\
- Existên \\
início e controlo do processo \\
- Formulação clara de metas e objetivos \\
- Consciência de que os benefícios não \\
são equititáveis \\
- Consciência de que os benefícios \\
- Legperados podem não ser atingidos \\
Legitimação
\end{tabular} & $\begin{array}{l}\text { Inibidores } \\
\text { - Envolvimento de stakeholders em função das suas necessidades } \\
\text { - } \text { Despoletar conflitos latentes entre stakeholders } \\
\text { - Competição pelos mesmos recursos } \\
\text { - Seleção inapropriada de stakeholders } \\
\text { - } \text { Dif́́cil sistematização dos processos (diferenças entre } \\
\text { nações/regiões/locais) } \\
\text { - } \text { Aumento do tempo para a concretização do projeto } \\
\text { - Cultura (política, local, administrativa) } \\
\text { - Dificuldades económicas das populações } \\
\text { - } \text { Falta de formação e experiência (dos locais, dos promotores) } \\
\text { - Implementação dificil em áreas com dificuldades ao nível do } \\
\text { planeamento e gestão das atividades recreativas e turísticas }\end{array}$ \\
\hline
\end{tabular}

Devem ser tidos em conta durante o processo

\begin{tabular}{|c|c|}
\hline \begin{tabular}{l}
\multicolumn{1}{c}{ Benefícios } \\
- Aumento da eficiência e eficácia dos processos \\
- Plano afetos às necessidades locais \\
- Soluções de baixo custo \\
- Agrupamento de recursos \\
- Oportunidades de formação e educação \\
- Aumento da capacitação local \\
- Partilha de custos \\
- Conhecimento social como complemento às \\
- políticas em desenvolvimento
\end{tabular} & $\begin{array}{l}\text { - Problemas } \\
\text { - Aumento do dispêndio financeiro (entidade promotora do processo) } \\
\text { - Risco do processo assumir direções em função dos interesses } \\
\text { - Benefícios podem não alcançar todos os envolvidos da forma } \\
\text { esperada } \\
\text { - Aumento das aspirações das populações (desânimo em caso de } \\
\text { - Dalha) } \\
\text { - } \text { - Fatores relacionais entre stakeholders } \\
\text { - Aumento do staff necessário para implementação do projeto } \\
\text { - Falta de consenso }\end{array}$ \\
\hline
\end{tabular}

Fonte: Elaboração própria.

4. O envolvimento e legitimação de stakeholders no processo: aspetos intrínsecos e normativos

Como referido anteriormente, um stakeholder no setor do turismo é aquele que tem o direito e a capacidade de participar e que, consequentemente é impactado pelo desenvolvimento do turismo de forma positiva ou negativa. Consideramos que esta aproximação inclusiva sugere a existência de algumas problemáticas, relacionadas com a qualificação e a decisão. Isto quer dizer que a capacidade de opinar num processo nem sempre está relacionada com a experiência para se formalizar ou efetivar essa mesma visão/opinião.

Os autores Khadka e Nepal (2010) referem que a experiência em vários projetos de conservação participativa mostra muitos constrangimentos nas fases de planeamento e implementação e que a identificação e inclusão apropriada de diferentes grupos específicos (stakeholders) para os programas de desenvolvimento e conservação tem sido uma das grandes falhas verificadas.

No planeamento do turismo, as possibilidades de colaboração são complicadas devido à existência de múltiplas e variadas organizações que possuem diferentes pontos de vista e diferentes interesses. Em destinos turísticos emergentes onde os interesses não estão coletivamente organizados, a identificação de stakeholders legítimos é uma tarefa complicada (Reed, 1997). De acordo com alguns autores, uma parte crítica e complexa da implementação de um planeamento colaborativo é a identificação e legitimação de potenciais stakeholders (Ladkin \& Bertramini, 2002; Roberts \& Simpson, 1999). Estas podem estar relacionadas com o seu poder económico ou político, a perceção legítima das revindicações, a urgência das mesmas e a vontade de estar envolvido (Medeiros de Araujo \& Bramwell, 1999). Em concordância com os autores, Gray (2004) assume que tentar promover a colaboração em processos de caráter ambiental pode ser extremamente difícil. Estas dificuldades surgem devido a diferentes níveis de jurisdição (e.g. governos locais, regionais e nacionais), diferenças no conhecimento e experiência de stakeholders, confrontos de valores, desconfiança histórica e frequente incerteza acerca da viabilidade dos resultados previstos.

Gray (2004) defende também que apesar de muitos trabalhos incidirem sobre fatores processuais para explicar os problemas entre stakeholders, a ênfase deve ser dada à convergência ou divergência da concetualização de conflito entre estes de forma a 
explicar o sucesso ou falhanço do processo colaborativo. Por outras palavras, esta concetualização refere-se à forma como os stakeholders compreendem o próprio conflito.

Uma forma clara de explicar esta assunção é dada pela mesma autora num trabalho desenvolvido nos finais dos anos 80 (Gray, 1989). Quando os stakeholders se envolvem numa parceria entre várias organizações fazem-no por duas razões distintas: 1) Para capitalizar através do trabalho conjunto e comum entre outros potenciais parceiros ou 2) numa tentativa de resolver propósitos de conflito. No entanto, na prática, estas duas motivações podem facilmente ocorrer interlaçadas. Se uma parceria se inicia com o objetivo de promover um objetivo comum, os stakeholders podem ver-se a si próprios em conflito sobre as ações que são necessárias na implementação dos planos que são propostos. No desenvolvimento metodológico do seu trabalho, Gray (2004) considerou algumas dimensões para compreender a interpretação dos stakeholders acerca do conflito gerado na área em estudo, nomeadamente: 1) Identidade, 2) caracterização, 3) gestão do conflito e 4) formas de ver/entender a natureza. A 1) identidade tenta capturar as formas como os stakeholders se descrevem a si próprios em termos do seu grupo social. Estes grupos tornam-se parte da sua autoidentidade e são refletidos nas respostas/assunções que estes fazem acerca de quem são; a 2) caracterização reflete a forma como os indivíduos ou grupos se vêm uns aos outros e captura alguns estereótipos negativos; a 3) gestão do conflito reflete as preferências dos stakeholders acerca da forma como o conflito deve ser resolvido; a 4) forma como entendem/vêm a natureza descreve as suas crenças acerca da proteção e regeneração da natureza.

No mesmo sentido, também os autores Donaldson e Preston (1995), num trabalho em torno da teoria de stakeholders, desenvolveram acerca de três aspetos destas teorias, nomeadamente: 1) descritivos/empíricos; 2) instrumentais e 3) normativos. O primeiro, é utilizado para descrever algumas características e/ou comportamentos da organização (ou individuo). Este aspeto é utilizado para examinar o estado passado, o presente e o futuro, dos negócios de uma determinada organização (análoga à dimensão “identificação" proposta por Gray).

O segundo, o aspeto instrumental, é utilizado para identificar conexões, ou a falta destas, entre stakeholders (análoga às dimensões "caracterização" e "gestão do conflito", proposta por Gray). $\mathrm{O}$ aspeto normativo é o núcleo fundamental da teoria de stakeholders. Este é utilizado para interpretar a função da corporação, incluindo a identificação moral ou filosófica pela qual determinada organização se guia (análoga à última dimensão proposta por Gray).

Assim, é importante ter uma clara noção de que nem todos os potenciais stakeholders têm de estar igualmente envolvidos num determinado processo de tomada de decisão. Igualmente importante é a noção de que não basta existirem um conjunto de características positivas (e.g. poder económico; experiência; entre outros). É fundamental que todos os interesses, perfis e padrões normativos sejam identificados e compreendidos. Em alguns trabalhos analisados, é feita referência ou esta complementaridade não é observada e pode constituir uma importante fraqueza no desenvolvimento de processos participativos (Arnaboldi \& Spiller, 2011; Bouwen \& Taillieu, 2004; Nistoreanu, Dorobantu, \& Tuclea, 2011; Stringer et al., 2006; Xu, Lü, Chen, \& Liu, 2009) . Em suma, a falha na identificação do perfil dos stakeholders pode determinar o insucesso do desenvolvimento do processo.

Para além das quatro dimensões de Gray (2004), outros fatores como o interesse no processo, o poder económico, a experiência ou os níveis de conhecimento foram também já referidos e são enfatizados em alguns trabalhos de Xu, Lü, Chen e Liu (2009), Ladkin e Bertramini, (2002), Medeiros de Araujo e Bramwell (1999), Miller, Rathouse, Scarles, Holmes, e Tribe (2010) e Bruyere, Beh, e Lelengula (2009). Outras variáveis são apresentadas no trabalho de Khadka e Nepal (2010), como o nível e escopo da participação local, as barreiras que inibem as pessoas de participarem e a perceção local de benefícios da sua própria participação em programas comunitários. Numa metodologia mais recente, Lee (2012) explorou variáveis latentes como o sentimento de envolvimento na comunidade, a afinidade, os custos e os benefícios percebidos, como influenciadores do apoio e envolvimento das comunidades no desenvolvimento do turismo sustentável.

Para além disso, é importante considerar que, associados à legitimação, devem estar subjacentes as diferenças entre o poder, a vontade e o interesse em participar, visto que por vezes não apresentam congruência.

É assim fundamental a compreensão de que não são unicamente os fatores processuais que determinam o sucesso/insucesso de um processo de planeamento e gestão participativa. Um passo prévio fundamental reside num conhecimento mais profundo de cada um dos potenciais participantes neste processo. Utilizamos a denominação "profundo" porque este conhecimento não se deve limitar a variáveis como o poder económico ou o nível educacional dos intervenientes. Deve existir uma transposição para um lado mais moral, que reflete, no caso da gestão turística e recreativa em $\mathrm{AP}$, a forma como cada stakeholder, entende este processo, os outros stakeholders, o setor do turismo e da recreação e o próprio meio natural.

Analisada a importância das dimensões intrínsecas e normativas inerentes aos processos de legitimação, e no sentido da especificidade, apresentamos no ponto seguinte um conjunto de fatores de informação a ter em conta aquando da iniciação de processos de gestão participativos e que devem envolver a participação ativa de todos os stakeholders envolvidos.

\subsection{Stakeholders como fonte de informação para a tomada de decisão}

De acordo com Garrod (2003) um importante papel que os stakeholders podem desempenhar em cada estado do ciclo de vida de determinado projeto é na recolha de informação que será necessária de forma a tomar decisões acerca do planeamento e gestão dos processos associados à recreação e ao turismo. A informação que é tipicamente necessária e que deve ser recolhida junto das diversas partes interessadas é inventariada no quadro seguinte (Quadro 1, sob a forma de questões). 
Quadro 1 - Fatores de informação

Qual o estado do espaço utilizado para o turismo (o "espaço" referese, por exemplo, a uma AP)

Quais as atividades de turismo e recreação desenvolvidas atualmente no espaço

Quais os impactos das atividades

Quais os impactos derivados de outras atividades (diretos ou indiretos)

Como e quão bem são protegidos os recursos

Quais os recursos que requerem mais e melhor proteção

Qual o real objetivo de ser desenvolverem certas formas de turismo

(e.g. ecoturismo)

Quem são os principais grupos de stakeholder presentes no espaço

Que aspirações e preocupação tem a população local relativamente

do desenv. Do turismo na área

Qual a consciência ecológica dos grupos de comunidades envolvidos no processo

Que iniciativas de turismo existem atualmente

Quão bem, determinado planeamento e legislação existente tem em conta o turismo ou a recreação no espaço

Qual a experiência passada da comunidade local nos processos de planeamento e gestão destas atividades

Quem são os líderes locais

Fonte: Adaptado de Garrod (2003).

Os âmbitos de alguns trabalhos científicos atuais respondem claramente às questões colocadas por Garrod (2003) e têm-se debruçado sobre diversas tipologias de stakeholders. Desde visitantes a organizações, diversas tipologias têm sido questionadas acerca da aceitação e proposta de novas opções de gestão (Randle \& Hoye, 2016; Smyth, Watzin, \& Manning, 2009), acerca do entendimento e aceitação da implementação de AP e das vantagens do desenvolvimento do turismo nestas (Haukeland, 2011), acerca dos benefícios económicos gerados pelo turismo ( $\mathrm{Xu}$ et al., 2009), as procuras dos turistas por infraestruturas (Haukeland, Grue, \& Veisten, 2010), problemas ambientais (Puczkó \& Rátz, 2000), problemas sociais (Mann \& Absher, 2008) entre outros.

A recolha de informação está diretamente relacionada com o campo instrumental e metodológico. Neste escopo, um vasto leque de trabalhos científicos baseia a sua recolha de informação numa vertente sobretudo interpretativa. A utilização de entrevistas semiestruturadas tem sido um dos instrumentos de recolha de dados mais evidenciados, sobretudo ao nível da consulta a organizações, ou informantes chave (Bruyere et al., 2009; Gray, 2004; McCool \& Guthrie, 2001; Miller et al., 2010; Raymond \& Hall, 2008). A vertente quantitativa tem como alvo principal as comunidades locais e grandes grupos de stakeholders.

\section{Conclusões}

Face ao exposto até ao momento, apresentamos a Figura 2 que reflete a relação entre os diversos pressupostos teóricos até então analisados, relacionando-os e gerando uma visão mais completa dos aspetos que devem ser considerados aquando da iniciação de processos de gestão participativos e dos seus participantes em particular (os stakeholders) relacionados com o turismo e a receação em AP. Considerando a realidade conhecida sobre as AP portuguesas e com base nos trabalhos de Rosa (2014) e Laranjo (2011), sugerem-se igualmente um conjunto alargado de stakeholders passíveis de serem inseridos/envolvidos nestes processos. O modelo de compreensão aqui apresentado conjuga os fundamentos referidos anteriormente com exemplos de tipologias de stakeholders e as variáveis comumente mais estudadas.

Em primeiro lugar, a figura representa um processo sequencial que deve estar associado à recolha de informação com vista à iniciação de processos participativos. Neste sentido, (caixa de texto na parte superior direita da figura) encontram-se os aspetos relacionados com a perceção dos stakeholders acerca das condições para o arranque do processo e dos benefícios esperados e, simultaneamente, a sua perceção sobre os aspetos que inibem a participação e os problemas potencialmente esperados. Estes aspetos, sobre os quais discorremos no início do artigo, são fundamentais para garantir o envolvimento dos stakeholders desde as fases mais embrionárias dum processo desta natureza, aspeto que potencia o sucesso do desenvolvimento sustentável do turismo (Waligo, Clarke, \& Hawkins, 2015).

Em segundo, a valorização de um conhecimento prévio das características dos stakeholders, de natureza mais tangível (caixa de texto abaixo do diagrama circular) e que incluem aspetos como o conhecimento, competências ou atividades desenvolvidas por estes e que podem ser úteis para a implementação de ações ao longo do processo. O conhecimento destas características surge a par com um conhecimento das dimensões intrínsecas destes indivíduos, valorizando-se aqui aspetos normativos e morais e onde se despistam eventuais situações de conflito. Estes fatores, se verificados num sentido positivo, podem potenciar o envolvimento (Sher, Bagul, \& Din, 2015).

Defendemos que é a conjugação destes dois grandes conjuntos de informação que permitem caracterizar os stakeholders e assim determinar o seu envolvimento no processo, contribuindo assim para uma legitimação mais fundamentada.

É igualmente importante denotar, que as tipologias de stakeholders apresentadas são constituídas por dois níveis: i) Um nível exterior (fora do círculo cinzento), composto essencialmente por entidades públicas administrativas com fortes influências nas políticas associadas às $\mathrm{AP}$, ao desporto e ao turismo; ii) Num nível interno (dentro do círculo cinzento), encontramos possibilidades de stakeholders, que podem logicamente variar em função da realidade da AP em estudo. Este conjunto alargado de tipologias $\mathrm{e}$ sua distinção a dois níveis é apresentada no trabalho de (Bello, 2016). É destas, visto que são em maior número e que a sua diversidade institucional pode também originar diversidade $e$ disparidade de perfis, ideais e filosofias, que derivam as dimensões intrínsecas, que no nosso entender, embora não tenham de ser exclusivas destes grupos tornam mais pertinente a sua compreensão.

A componente inferior da figura considera ainda as dimensões de informação que normalmente são procuradas junto dos stakeholders e que são essenciais para a obtenção de informação pertinente para a gestão e tomada de decisão.

Num sentido conclusivo, consideramos que aspetos como a comunicação, a perceção comum de benefícios e problemas à implementação de processos participativos, a existência de uma estratégia comum desde os níveis mais elevados aos mais baixos 
da cadeia participativa, uma noção clara da importância, da função e dos potenciais benefícios e problemas derivado do turismo e da recreação em AP, são alguns dos fatores mais importantes a ter em consideração no arranque de um processo de planeamento e gestão participativa.

Figura 2 - Stakeholders do turismo e recreação em áreas protegidas

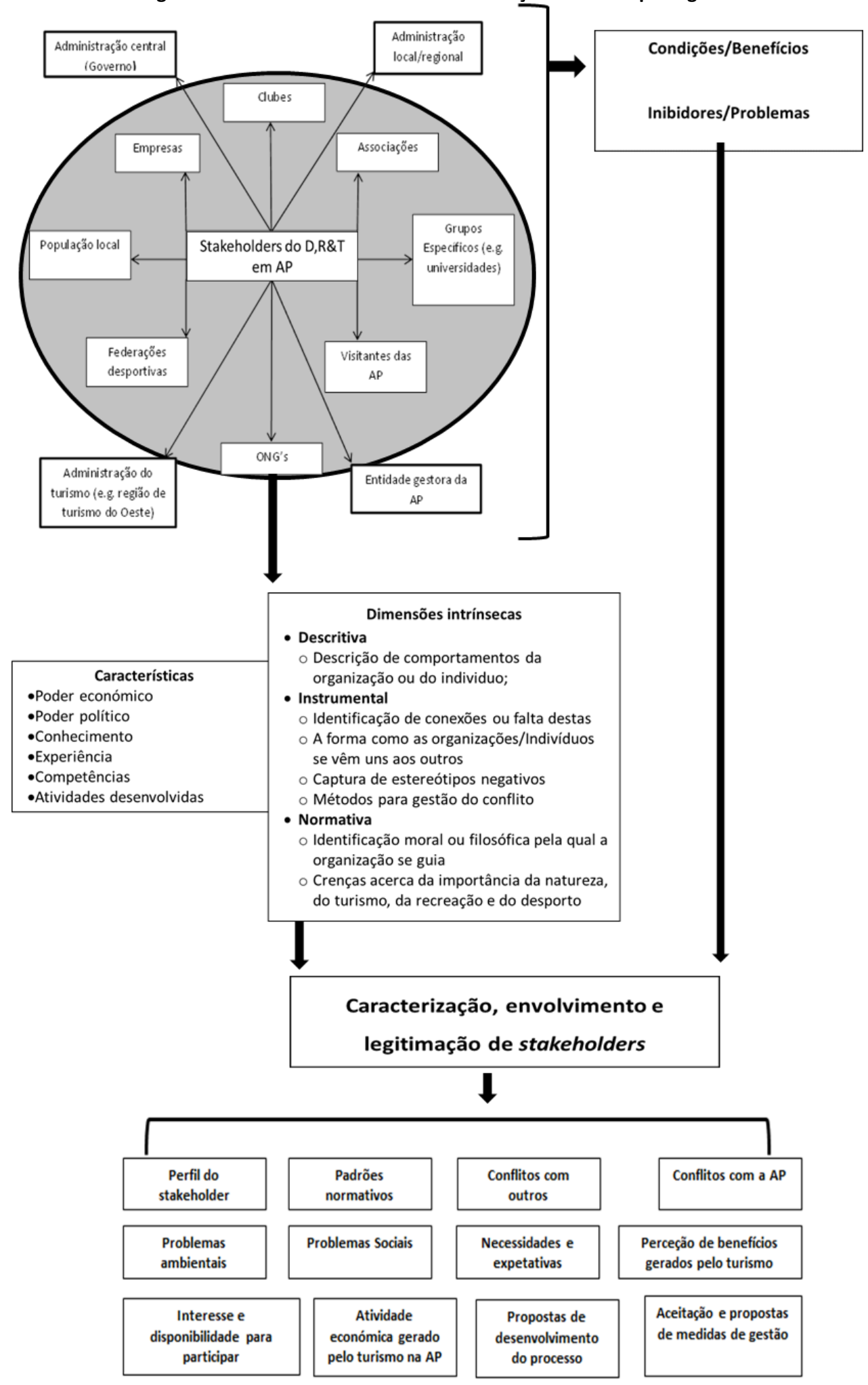

Fonte: Elaboração própria.

Face aos resultados avaliados nos diferentes estudos, reforçados com a assunção de McComb e Boyd (2016), a consideração de elementos unicamente processuais baseados em documentos orientadores, estruturas burocráticas e definições estanques de regras e objetivos são, do nosso ponto de vista, considerações que tidas em conta isoladamente ditam o fracasso de um processo que envolve pessoas e comunidades normalmente rurais e de reduzida dimensão. 
Uma compreensão mais ampla do perfil dos stakeholders e das suas perceções aos mais variados níveis, revela-se na literatura, um fator fundamental para o arranque do processo. Ao utilizarmos a palavra "arranque" queremos dizer que esta compreensão deve decorrer antes do próprio processo em si, e os seus resultados, devem ser essenciais para determinar o encaminhamento ou não dessa estratégia de cooperação.

É nesse sentido que, novos estudos a realizar nas AP nacionais, associados aos setores da recreação e o do turismo, tenham em conta a importância efetiva dos diferentes stakeholders. Igualmente, considerando a possibilidade de se desenvolverem trabalhos sobre a temática especifica da gestão destes espaços, é fundamental que não se assuma como garantida a efetividade e adequabilidade de uma abordagem participativa (pelos motivos morais associados à sua fundamentação) sem que um conhecimento prévio e multidimensional dos diversos stakeholders seja considerado.

\section{Referências}

Aas, C., Ladkin, A., \& Fletcher, J. (2005). Stakeholder Collaboration and Heritage Management. Annals of Tourism Research, 32(1), 28-48. doi: 10.1016/j.annals.2004.04.005.

Arnaboldi, M., \& Spiller, N. (2011). Actor-network theory and stakeholder collaboration: The case of Cultural Districts. Tourism Management, 32, 641-654.

Bello, F. (2016). Enhancing community participation in tourism planning associated with protected areas in developing countries: Lessons from Malawi. Tourism and Hospitality Research, (Published online ahead of print), doi: 10.1177/1467358416647763.

Borrini-Feyerabend, G., Dudley, N., Jaeger, T., Lassen, B., Broome, N., Phillips, A., \& Sandwith, T. (2013). Governance of Protected Areas: From understanding to action. Gland: IUCN. Consultado em 20 janeiro 2016 em http://cmsdata.iucn.org/downloads/

governance_of_protected_areas_from_understanding_to_action.pdf.

Bouwen, R., \& Taillieu, T. (2004). Multi-party collaboration as socia learning for interdependence: developing relational knowing for sustainable natural resource management. Journal of Community \& Applied Social Psychology, 14(3), 137-153.

Bramwell, B., \& Lane, B. (2000). Tourism Collaboration and Partnerships: Politics, Practice and Sustainability. Cleveland: Channel View.

Bruyere, B., Beh, A., \& Lelengula, G. (2009). Differences in Perceptions of Communication, Tourism Benefits, and Management Issues in a Protected Area of Rural Kenya. Environmental Management(43), 49-59. doi: 10.1007/s00267-008-9190-7.

Byrd, E. (2003). An Analysis of Variables that Influence Stakeholder Participation and Support for Sustainable Tourism Development in Rural North Carolina. PhD, Graduate Faculty of North Carolina State University, North Carolina.

Byrd, E. (2007). Stakeholders in sustainable tourism development and their roles: Applying stakeholder theory to sustainable tourism development. Tourism Review, 62(2), 6-13

Dearden, P., Bennett, M., \& Johnston, J. (2005). Trends in global protected area governance, 1992-2002. Environmental Management, 36(1), 89-100.

Donaldson, T., \& Preston, L. (1995). The Stakeholder Theory of the Corporation: Concepts, Evidence, and Implications. The Academy of Management Review, 20(1), 65-91.

Eagles, P. (2009). Governance of recreation and tourism partnerships in parks and protected areas. Journal of Sustainable Tourism, 17(2), 231-248. doi: 10.1080/09669580802495725.

Garrod, B. (2003). Local Participation in the Planning and Management of Ecotourism: A Revised Model Approach. Journal of Ecotourism, 2(1), 33-53. doi: 10.1080/14724040308668132.

Gray, B. (1985). Conditions facilitating interorganizational collaboration Human Relations, 38(10), 911-936. doi: 10.1177/001872678503801001.
Gray, B. (1989). Collaborating: Finding Common Ground for Multiparty Problems. San Francisco: Jossey-Bass.

Gray, B. (2004). Strong Opposition: Frame-Based Resistance to Collaboration. Journal of Community and Applied Social Psychology(14), 166-176. doi: 10.1002/casp.773.

Hall, C. (2000). Tourism Planning Processes and Relationships. Harlow: Prentice Hall.

Haukeland, J. (2011). Tourism stakeholders' perceptions of nationla park management in Norway. Journal of Sustainable Tourism, 19(2), 133-153. doi: 10.1080/09669582.2010.517389.

Haukeland, J., Grue, B., \& Veisten, K. (2010). Turnin National Parks into Tourist Attractions: Nature Orientation and Quest for Facilities. Scandinavian Journal of Hospitality and Tourism, 10(3), 248-271. doi: 10.1080/15022250.2010.502367.

Jamal, T., \& Getz, D. (1995). Collaboration Theory and Community Tourism Planning. Annals of Tourism Research, 22(1), 186-204.

Kalamandeen, M., \& Gillson, L. (2007). Demything "wilderness": implications for protected area designation and management. Biodiversity Conservation, 16, 165-182. doi: 10.1007/s10531-006-9122-x.

Khadka, D., \& Nepal, S. (2010). Local Responses to Participatory Conservation in Annapurna Conservation Area, Nepal. Environmenta Management, 45(2), 351-362. doi: 10.1007/s00267-009-9405-6.

Ladkin, A., \& Bertramini, A. (2002). Collaborative Tourism Planning: A Case Study of Cusco, Peru. Current Issues in Tourism.

Laranjo, J. (2011). A Gestão do Turismo de Natureza na Rede Nacional de Áreas Protegidas: Mestrado, Universidade Nova de Lisboa, Lisboa.

Lee, T. (2012). Influence analysis of community resident support for sustainable tourism development. Tourism Management, xxx, 1-10.

Mann, C., \& Absher, J. D. (2008). Recreation conflit potential and management implications in the northern/central Black Forest Natura Park. Journal of Environmental Planning and Management, 51(3), 363-380. doi: 10.1080/09640560801979527.

McComb, E., \& Boyd, S. (2016). Stakeholder collaboration: A means to the success of rural tourism destinations? A critical evaluation of the existence of stakeholder collaboration within the Mournes, Northern Ireland Tourism and Hospitality Research, February,2(Published online before print).

McCool, S., \& Guthrie, K. (2001). Mapping the Dimensions of Successfu Public Participation in Messy Natural Resources Management Situations. Society and Natural Resources(14), 309-323. doi: 0894-1920/2001.

Medeiros de Araujo, L., \& Bramwell, B. (1999). Stakeholder Assessment and Collaborative Tourism Planning: The Case of Brazil's Costa Dourada Project. Journal of Sustainable Tourism, 7(3\&4), 356-378. doi: 09669582/99/03 0356-23.

Miller, G., Rathouse, K., Scarles, C., Holmes, K., \& Tribe, J. (2010). Public Understanding of Sustainable Tourism. Annals of Tourism Research, 37(3), 627-645. doi: 10.1016/j.annals.2009.12.002

Nistoreanu, P., Dorobantu, R., \& Tuclea, E. (2011). Strategic Directions in Sustainable Tourism Development Through Rural Tourism Activities. Forum Ware International (Special issue)(1), 116-122.

Patti, S. (2013). Sustainability and Support for the Ecotourism within Etna Park Area. American Journal of Tourism Research 2(1), 124-129.

Pimbert, M., \& Pretty, J. (1995). Parks, People and Professionals: Putting 'Participation' into Protected Area Management (pp. 65). Geneva: United Nations Research Institue for Social Development

Pinto, B. (2008). Historical information of the Portuguese Protected Areas and its implications for management. PhD, Universidade Nova de Lisboa, Lisboa.

Puczkó, L., \& Rátz, T. (2000). Tourist and Resident Perceptions of the Physical Impacts of Tourism at Lake Balaton, Hungary: Issues for Sustainable Tourism Management. Journal of Sustainable Tourism, 8(6), 458-478. doi: 10.1080/09669580008667380.

Randle, E., \& Hoye, R. (2016). Stakeholder perception of regulating commercial tourism in Victorian National Parks, Australia. Tourism Management, 54, 138-149. 
Raymond, E., \& Hall, C. (2008). The Development fo Cross-Cultural (Mis) Understanding Through Volunteer Tourism. Journal of Sustainable Tourism, 16(5), 530-543.

Reed, M. (1997). Power relations and community-based tourism planning. Annals of Tourism Research, 24(3), 566-591. doi: 10.1016/S01607383(97)00023-6.

Roberts, L., \& Simpson, F. (1999). Developing Partnership Approaches to Tourism in Central and Eastern Europe. Journal of Sustainable Tourism, 7(3\&4), 314-330. doi: 0966-9582/99/03 0314-17.

Rosa, P. (2014). Desporto, Recreação e Turismo em Áreas Protegidas: Modelo de Gestão Participada para o Parque Natural das Serras de Aire e Candeeiros. PhD, Universidade da Madeira, Madeira.

Selin, S. (1999). Developing a Typology of Sustainable Tourism Partnerships. Journal of Sustainable Tourism, 7(3-4), 260-273. doi: 10.1080/09669589908667339.

Selin, S., \& Chavez, D. (1995). Developing a Collaborative Model for Environmental Planning and Management. Environmental Management, 19(2), 189-195.

Sher, K., Bagul, A., \& Din, S. (2015). The Influence of Community Attachment and Community Involvement Towards Resident's Support on Sustainable Tourism Development by Mediating Perceived Benefits and Perceived Costs. American-Eurasian J. Agric. \& Environ. Sci., 15, 133-138.

Smyth, R., Watzin, M., \& Manning, R. (2009). Investigating public preferences for managing Lake Champlain using a choice experiment. Journal of Environmental Management, 90(1), 615-623. doi: 10.1016/j.jenvman.2007.12.016.

Stringer, L., Dougill, A., Fraser, E., Hubacek, K., Prell, C., \& Reed, M. (2006). Unpacking "Participation" in the Adaptive Management of Socialecological Systems: a Critical Review. Ecology and Society, 11(2), 39.

Swarbrooke, J. (1999). Sustainable Tourism Management. Wallingford: CABI.

Timothy, D. (1999). Participatory Planning: A View of Planning in Indonesia. Annals of Tourism Research, 26(2), 371-391.

Tosun, C., \& Jenkins, C. (1998). The evolution of tourism planing in thirdworld countries: A critique. Progress in Tourism and Hospitality Research, 4(2), 101-114.

Waligo, V., Clarke, J., \& Hawkins, R. (2015). Embedding stakeholders in sustainable tourism strategies. Annals of Tourism Research, 55, 90-93.

Walpole, M., \& Goodwin, H. (2001). Local attitudes towards conservation and tourism around Komodo National Park, Indonesia. Environmental Conservation, 28(2), 160-166.

Wray, M. (2011). Adopting and implementing a transactive approach to sustainable tourism planning: translating theory into practice. Journal of Sustainable Tourism, 19(4-5), 605-627. doi: 10.1080/09669582.2011.566928.

Xu, J., Lü, Y., Chen, L., \& Liu, Y. (2009). Contribution of tourism development to protected area management: local stakeholder perspectives. International Journal of Sustainable Development \& World Ecology, 16(1), 30-36. doi: 10.1080/13504500902757189.

Yodsuwan, C., \& Butcher, K. (2012). Determinants of Tourism Collaboration Member Satisfaction in Thailand. Asia Pacific Journal of Tourism Research, $17(1), 63-80$.

Recebido: 25.02.2015

Reenviado: 19.07.2016

Aceite: 25.07.2016 